\title{
Finite One-Dimensional Photonic Crystal with Gaussian Modulation: Transmission and Escape
}

\author{
María de la Luz Silba-Vélez ${ }^{*}$, David-Armando Contreras-Solorio'1, Rolando Pérez-Álvarez², \\ Carlos Iván Cabrera ${ }^{1}$ \\ ${ }^{1}$ Academic Unit of Physics, Autonomous University of Zacatecas, Zacatecas, Mexico \\ ${ }^{2}$ Center of Science Research, Institute of Basic and Applied Sciences, Autonomous University of the State of Morelos, Cuernavaca, \\ Mexico \\ Email: *madelaluzsilbavelez@outlook.com,dacs10@yahoo.com.mx, rpa@uaem.mx, carlos.cabrera@fisica.uaz.edu.mx
}

How to cite this paper: de la Luz SilbaVélez, M., Contreras-Solorio, D.-A., PérezÁlvarez, R. and Cabrera, C.I. (2017) Finite One-Dimensional Photonic Crystal with Gaussian Modulation: Transmission and Escape. Optics and Photonics Journal, 7, 170-180.

https://doi.org/10.4236/opj.2017.710017

Received: September 15, 2017

Accepted: October 27, 2017

Published: October 30, 2017

Copyright (C) 2017 by authors and Scientific Research Publishing Inc. This work is licensed under the Creative Commons Attribution International License (CC BY 4.0).

http://creativecommons.org/licenses/by/4.0/

\begin{abstract}
This paper studied the transmission coefficient and escape frequencies in a system of planar dielectric layers where the refractive index changes from one layer to another through a Gaussian function. The wave equation with normal incidence is analyzed. For the calculations, the transfer matrix formalism is used. In a previous work, the transmission and escape problem for Gaussian electronic superlattices is investigated. Now it studied the electromagnetic modes for a system formed by layers where the refractive index of the structure is modulated by a Gaussian function. The system presents transparency bands of transmission and gaps without transmission. The escape frequencies $\omega=\omega_{r}-i \Gamma$ are situated near these transparency bands but they do not coincide with them. $\omega_{r}$ is the frequency (mode) and $\Gamma$ describes the width of the states. For these systems, the escape states are very wide. A non Gaussian system presents resonance peaks in the transmission and the escape states are narrow. The formation of transparency bands in the transmission for a Gaussian system is attributed to the widening of the escape states.
\end{abstract}

\section{Keywords}

Transmission Coefficient, Escape Frequencies, Photonic Crystal

\section{Introduction}

When we talk about photonic crystals, we refer to periodic structures as systems used to manipulate light. At the 80's years, Yablonovich and Sajeev proposed to make artificial structures with dielectric material for the study of the electromagnetic properties of crystal photonics. The first one was interested in inhibiting spontaneous emission, while the second was interested in the study of 
light introducing a small localized disorder in the periodic system [1] [2]. Knowledge of the optical properties of materials is a factor that impacts the technological progress, for example in the construction of devices or telecommunications because of the possibility of being able to build such systems today [3] [4]. Nowdays we can build structures with dielectric materials like wave guides, mirrors, microcavities or passband filters [5].

The study for energy, electronic, optical and acoustic filters is an interesting and active field. Formerly frequency band pass filters where light can be transmitted in full when the frequency of the incident photons corresponds to the passband and total reflection occurs when the incident energy corresponds to the stopband, were studied. For example, the study of periodic profile systems, i.e. by bilayers formed with refractive indices $n_{1}$ and $n_{2}$ systems correspondingly [6] [7]. We focused on a little more interesting systems. We study the transmission and escape frequencies in a photonic crystal where the refractive index of the structure are modulated by the Gaussian function as proposed in [8]. Such systems have been studied and it has been observed that the probability of transmission is almost equal to unity in the passband. We also show results in regular systems, i.e., layers with only two values of refractive indices $n_{1}$ and $n_{2}$. In regular systems there are transmissions bands which present resonance peaks inside the bands with high values of transmittance but there is not transparency bands.

On the other hand, we mean by escape frequencies the resonance frequencies for an open system which is separated by the exterior by a partly transparent boundary surface. This structure losses energy to the exterior via radiation and we do not consider radiation coming into the system from the exterior. In this case the frequencies obtained are complex eigenvalues. The formation of transparency bands in a Gaussian system is an outstanding fact. We think that these transparency bands are due to the smoothness of the Gaussian change of refractive index which facilitates the transmission of the waves. On the other hand, this kind of variation of refractive index, with high value of index in the middle of the structure and progressive reduction to the extremes of the system, would make the waves to escape more easily from the structure. This means that there would be a reduction of the states lifetime and a widening in frequency. Then, the transparency bands would be the envelope of the wide escape states. Thus, our purpose in this work is to compare the transparency bands with the escape states.

For electromagnetic waves, in [9], they found that in case of 1D Fabry-Perot structure the transmission resonance frequencies and real parts of the complex eigenfrequencies are identical but if a multilayer system is considered the values of the escape frequencies are different from the frequencies resonance for the transmittance, although they may be very close [10]. We found in previous work where the electronic problem was studied [11] that the escape energies are different from the energy resonances in the transmittance, and this is much more pronounced for Gaussian superlattices (where the barriers height is 
modulated by a Gaussian function) than for regular ones where all the barriers have the same height. The analogy between electrons in semiconductor materials and photons in photonic crystals allows us to use the same methodology to calculate the coefficient of transmission and study the escape problem through the transfer matrix formalism [12] [13] [14] [15]. In this case, we change the type of matrix due to some numerical limitations of the associated transfer matrix used in the previous work. We follow the same idea to the Schrödinger equation [11] but now applied to the Maxwell equation.

The paper is organized as follows. In Section 2, we present the theoretical model. We use the transfer matrix formalism. In Section 3, we describe the structures we are interested in. In Section 4, we present our results. Finally, in Section 5 we formulate our conclusions.

\section{Theoretical Model}

We can follow the same idea as in [11] but now applied to the Maxwell equation. In fact, it is analogous, because the differential operator of the Maxwell equation is similar to the equation of Schrödinger with the difference in the characteristic parameters.

In the case of a polarization wave $s$ where the electric field vector $E$ is transverse to the plane of incidence can be determined by applying the conditions of continuity in the interface. All electric field are perpendicular to the incident plane, with outward direction of the sheet, while two magnetic field vectors are taken so that the flow energy is positive in the direction of propagation. We describe the propagation light with variable refractive index by the equation

$$
\frac{\mathrm{d}}{\mathrm{d} x}\left[\frac{\mu_{0}}{\mu(x)} \frac{\mathrm{d} E_{z}(x)}{\mathrm{d} x}\right]+\left[\omega^{2} \mu_{0} \varepsilon(x)-\frac{\mu_{0}}{\mu(x)} \kappa^{2}\right] E_{z}(x)=0,
$$

where $E_{z}(x)$ and $\frac{\mu_{0}}{\mu(x)} \frac{\mathrm{d} E_{z}(x)}{\mathrm{d} x}$ are continuous functions. The equation of motion is derived from the Maxwell equations in the substance,

$$
\begin{gathered}
\nabla \cdot \boldsymbol{D}(\boldsymbol{r}, t)=\rho(\boldsymbol{r}, t), \\
\nabla \times \boldsymbol{E}(\boldsymbol{r}, t)=-\frac{\partial \boldsymbol{B}(\boldsymbol{r}, t)}{\partial t}, \\
\nabla \cdot \boldsymbol{B}(\boldsymbol{r}, t)=0, \\
\nabla \times \boldsymbol{H}(\boldsymbol{r}, t)=\boldsymbol{j}(\boldsymbol{r}, t)+\frac{\partial \boldsymbol{D}(\boldsymbol{r}, t)}{\partial t} .
\end{gathered}
$$

If there are no sources, the fields are of the form

$$
\begin{aligned}
& \boldsymbol{E}_{t}(\boldsymbol{\rho}, x, t)=\boldsymbol{E}_{0 t}(x) \mathrm{e}^{i(\boldsymbol{\kappa} \cdot \rho-\omega t)}, \\
& \boldsymbol{H}_{t}(\boldsymbol{\rho}, x, t)=\boldsymbol{H}_{0 t}(x) \mathrm{e}^{i(\boldsymbol{\kappa} \cdot \boldsymbol{\rho}-\omega t)}, \\
& E_{z}(\boldsymbol{\rho}, x, t)=E_{0 z}(x) \mathrm{e}^{i(\boldsymbol{\kappa} \cdot \boldsymbol{\rho}-\omega t)},
\end{aligned}
$$




$$
H_{z}(\boldsymbol{\rho}, x, t)=H_{0 z}(x) \mathrm{e}^{i(\boldsymbol{\kappa} \cdot \boldsymbol{\rho}-\omega t)},
$$

with

$$
\begin{gathered}
\boldsymbol{\rho}=y \boldsymbol{e}_{y}+z \boldsymbol{e}_{z}, \\
\boldsymbol{\kappa}=\kappa_{y} \boldsymbol{e}_{y}+\kappa_{z} \boldsymbol{e}_{z}, \\
\boldsymbol{E}_{0 t}(x)=E_{0 y}(x) \boldsymbol{e}_{y}+E_{0 z}(z) \boldsymbol{e}_{z}, \\
\boldsymbol{H}_{0 t}(x)=H_{0 y}(x) \boldsymbol{e}_{y}+H_{0 z}(x) \boldsymbol{e}_{z} .
\end{gathered}
$$

With a little algebraic manipulation we can obtain (1) considering that $D_{x} \equiv 0$. We study one-dimensional systems where the profile for the refractive index $n_{\text {sys }}(x)$ is described as Section 3.

We concentrate in a structure of $N$ plane dielectric layers perpedicular to the $x$ axis. The width of each $j$ layer are denoted by $d_{j}$ and each refractive index by $n_{j}$. We also consider the study in medium homogeneous, isotropic and lossless. In other words, we consider a system where at its ends, the refractive indices are constant $\left(n_{L}\right.$ and $\left.n_{R}\right)$, while in the intermediate zone the refractive index $n(z)$ is dependent on the position (the growth direction of the heterostructure). We use the formalism of transfer matrix conformed by the dynamical matrix $D_{j}$ and the propagation matrix $P_{j} \quad[16]$.

We know that the energy flux is given by the Poynting vector $\boldsymbol{S}$. Imposing the condition of continuity in the interface $(x=0)$ for $E_{y}$ and $H_{z}$ we have

$$
\begin{gathered}
E_{1}+E_{1}^{\prime}=E_{2}+E_{2}^{\prime}, \\
\sqrt{\frac{\epsilon_{1}}{\mu_{1}}}\left(E_{1}-E_{1}^{\prime}\right) \cos \theta_{1}=\sqrt{\frac{\epsilon_{2}}{\mu_{2}}}\left(E_{2}-E_{2}^{\prime}\right) \cos \theta_{2} .
\end{gathered}
$$

$\theta_{1}$ and $\theta_{2}$ are the angles of the wave vectors $k_{1}$ and $k_{2}$, respectively, with respect to the normal to the interface. These two equations can be rewritten in matrix form as

$$
D_{1}\left(\begin{array}{c}
E_{1} \\
E_{1}^{\prime}
\end{array}\right)=D_{2}\left(\begin{array}{l}
E_{2} \\
E_{2}^{\prime}
\end{array}\right) \text {. }
$$

Then we can rewrite the continuity condition in $N$ layers for every interface through a matrix

$$
D_{j}=\left(\begin{array}{cc}
1 & 1 \\
\sqrt{\frac{\epsilon_{j}}{\mu_{j}}} \cos \theta_{j} & -\sqrt{\frac{\epsilon_{j}}{\mu_{j}}} \cos \theta_{j}
\end{array}\right) .
$$

$D_{j}$ is so-called dynamic matrix $s$ for $j=1,2, \cdots, N$.

Analogously we can the same methodology followed for polarization $p$ [8] [16]. In our case we only focus on the polarization $s$ since the study is at normal incidence. The objective of the coefficient matrix is to transfer the coefficients of the solutions of the field, in the manner

$$
\left[\begin{array}{l}
A_{0} \\
B_{0}
\end{array}\right]=\left[\begin{array}{ll}
M_{11} & M_{12} \\
M_{21} & M_{22}
\end{array}\right]\left[\begin{array}{l}
A_{s} \\
B_{s}
\end{array}\right],
$$


$A_{0}, B_{0}, A_{s}, B_{s}$ corresponding to coefficients incoming and outgoing coefficients media respectively. We define the matrix $P_{j}$ which is so-called propagation matrix, it considers the phase change due to the thickness of each layer

$$
P_{j}=\left[\begin{array}{cc}
\mathrm{e}^{-i \phi_{j}} & 0 \\
0 & \mathrm{e}^{i \phi_{j}}
\end{array}\right] .
$$

$\Phi_{j}$ is given by $\phi_{j}=k_{j} d_{j}=\frac{n_{j} \omega d_{j}}{c} \cos \theta_{j}$ where $k_{j}=\frac{n_{j} \omega}{c} \cos \theta_{j}$. It is possible to show that a layered system such as that shown in Figure 1. To make the total matrix of the system is given by

$$
D_{0}^{-1}\left[\prod_{i=1}^{N} D_{j} P_{j} D_{j}^{-1}\right] D_{s}=\left[\begin{array}{ll}
M_{11} & M_{12} \\
M_{21} & M_{22}
\end{array}\right] .
$$

The system is composed of Nlayers, where $D_{0}$ and $D_{s}$ refers to matrices of the corresponding incoming and outgoing media. Details can be seen in [16] [17].

We are interested in studying the scattering problem of (18). In this problem we consider the incoming, reflected and transmitted waves, then the coefficient $B_{s}$ is null. The transmittance $\mathrm{T}$ is given by the ratio of the Poynting power flow of the transmitted wave to that of the incidente wave, then

$$
T=\frac{n_{s}}{n_{0}}\left|\frac{1}{M_{11}}\right| .
$$

The reflection coefficient $R$ is obtained in a similar way, or taking into account that $R+T=1$.

The problem of escape is related to having photons confined in the crystal and they leave the system. We consider only outgoing waves, and the coefficients $A_{0}=0$ and $B_{s}=0$ out of the interval $\left(x_{0}, x_{s}\right)$. Then (18) gives the transcendental equation,

$$
M_{11}=0
$$

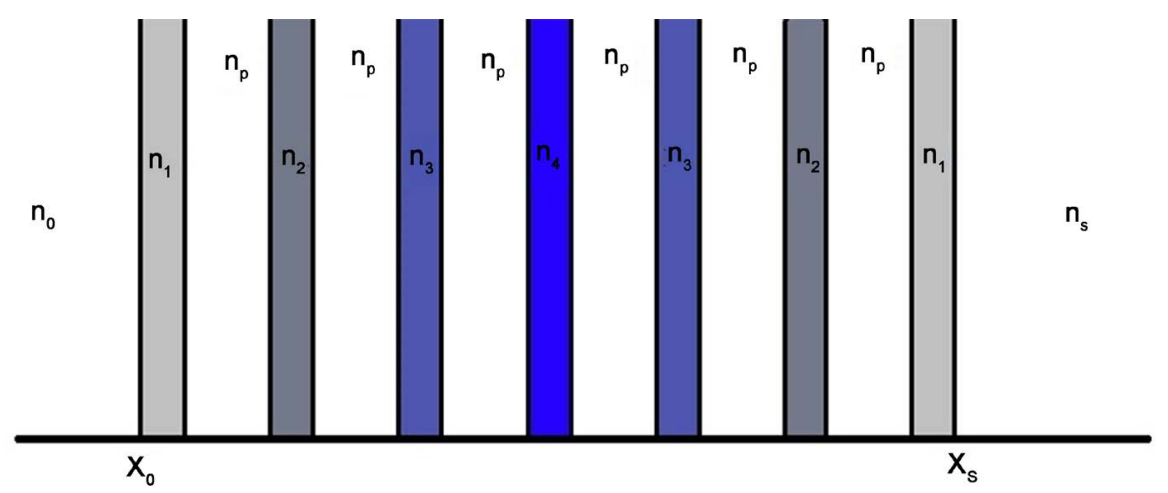

Figure 1. The general refractive index profile are modulated by a Gaussian function. $n_{1}=n_{\min } \cdot n_{4}=n_{\max }$. The medium of incidence has index $n_{0}$ and the medium after the structure has medium $n_{s}$. 
The solutions to (22) have the form $\omega=\omega_{r}-i \Gamma$, where the real part $\omega_{r}$ represents the frequency while the imaginary part $\Gamma$ describes the fact that the modes have finite lifetime and they decay. For simplicity we make a change of variable, in the Section 3 we will explain in detail.

\section{The Refractive Index Profile}

\subsection{System Modulated by a Gaussian Function}

We are interested in a photonic crystal where the refractive index of the structure is modulated by the Gaussian function (see Figure 1). We can use the proposal in [8], for odd layers $n(x)=\left(n_{\max }-n_{\min }\right) \exp \left(-\left(\left(x-x_{0}\right) / \sigma\right)^{2}\right)+n_{\min }$ where $n_{\max }$ is the refractive index of the value of the central layer, $n_{\min }$ the minimum value of the initial and final layer and $\sigma$ is the standard deviation. To guarantee that the initial and final layer take the value 1.2 we take

$$
n_{\min }=\frac{1.2-n_{\max } \exp (-x / \sigma)^{2}}{1-\exp \left(-(x / \sigma)^{2}\right)}
$$

We do this in order that the traveling wave traverses the structure in a smooth way as possible. In all cases studied we take $n_{\max }=2$. The even layers are constant and for our case we consider them with a value equal to the exterior of the structure. We show a result for system where the odd layers are modulated by a Gaussian function so that the maximum refractive index is at the ends and the minimum at the center of the structure to compare them (Section 4).

The calculations are performed at normal incidence with non-magnetic materials. The ratio of the widths of the layers $d_{\text {even }} / d_{\text {odd }}=8$. It was normalized in the frequency range and instead of plotting in function of $\omega$ the results are presented in function of $f \Delta / c$. In this case we consider the total width of the structure as $\Delta=1$ and $f$ is the frequency ${ }^{1}$.

\subsection{Regular System}

We refer to regular system to structures formed by refractive indices $n_{1}$ and $n_{2}$. The refractive indices are interleaved with odd number of layers $N_{c}=3,5,7, \cdots$. For example, for $N_{c}=7$ we have refractive indices $n_{1}, n_{2}, n_{1}, n_{2}, n_{1}, n_{2}, n_{1}$ and with its corresponding widths $d_{1}$ and $d_{2}$.

\section{Results and Discussion}

In this section we present the transmission coefficients and escape frequencies for systems in which the profiles are formed for layers modulated by a Gaussian function. We show it for 17, 25, 37, 45 layers with Gaussian modulation (see Figure 2 and Figure 3), the case reversed for 17 layers (see Figure 4) and the case regular also for 17 layers (see Figure 4). When we refer to the inverse ${ }^{1} \omega=2 \pi f$. 


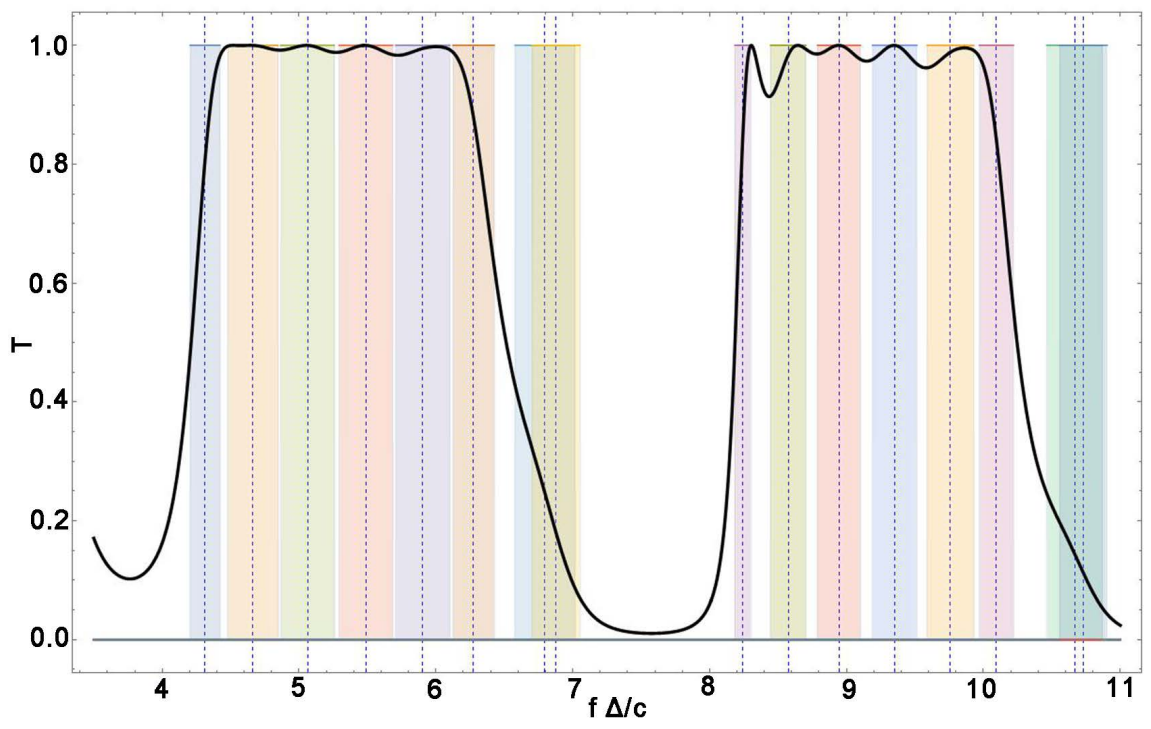

(a)

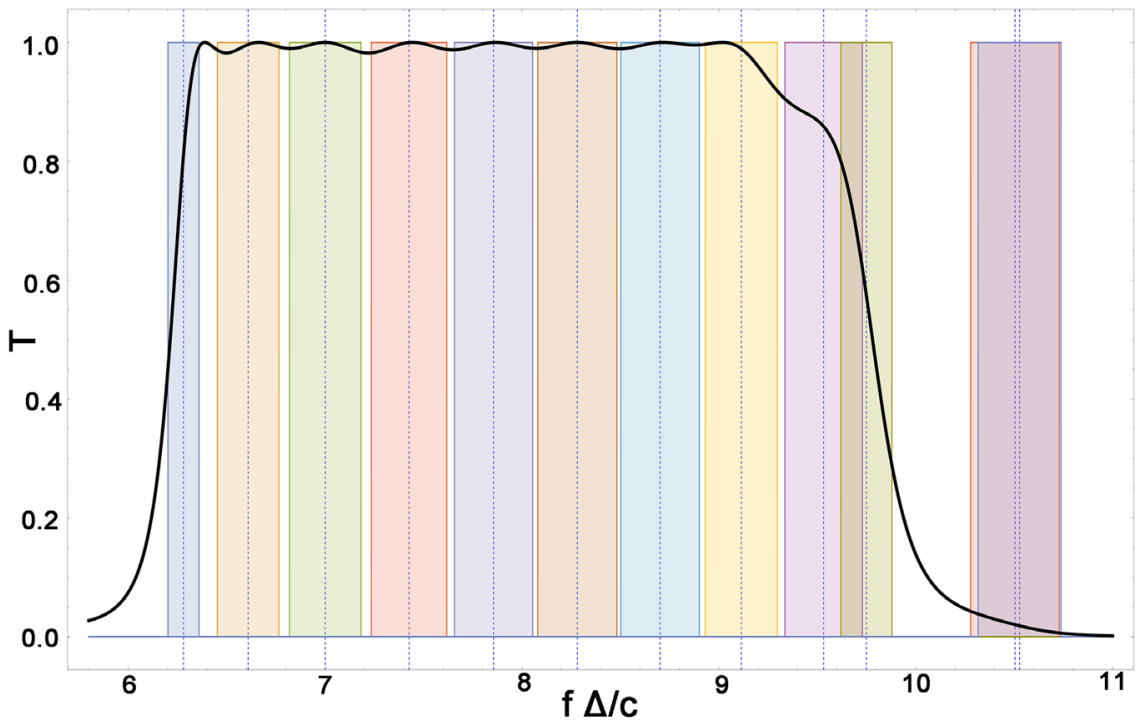

(b)

Figure 2. Transmission coefficient of for systems. The refractive index of odd layers of the structure are modulated by a Gaussian function. The parameters in such a system are: $n_{0}=n_{s}=n_{p}=1, n_{\max }=2$ and $d_{p}=8 d_{\text {odd }}$. The blue dashed lines correspond to the real part of the solution of the escape problem while the rectangles centered on each of them represent their corresponding $\Gamma$. (a) $n_{\min }=1.183$ and $N_{c}=17$; (b) $n_{\min }=1.184$ and $N_{c}=25$. We show in the upper curve (a) a gap of no transmission between two transparency bands.

system the odd layers are modulated by a Gaussian function so that the maximum refractive index is at the ends and the minimum at the center of the structure. The regular system case consist in that the value of the refractive index in the odd layers is constant and equal.

We use allowable values for porous silicon. We can observe that the greater the number of layers the passband is better defined. 


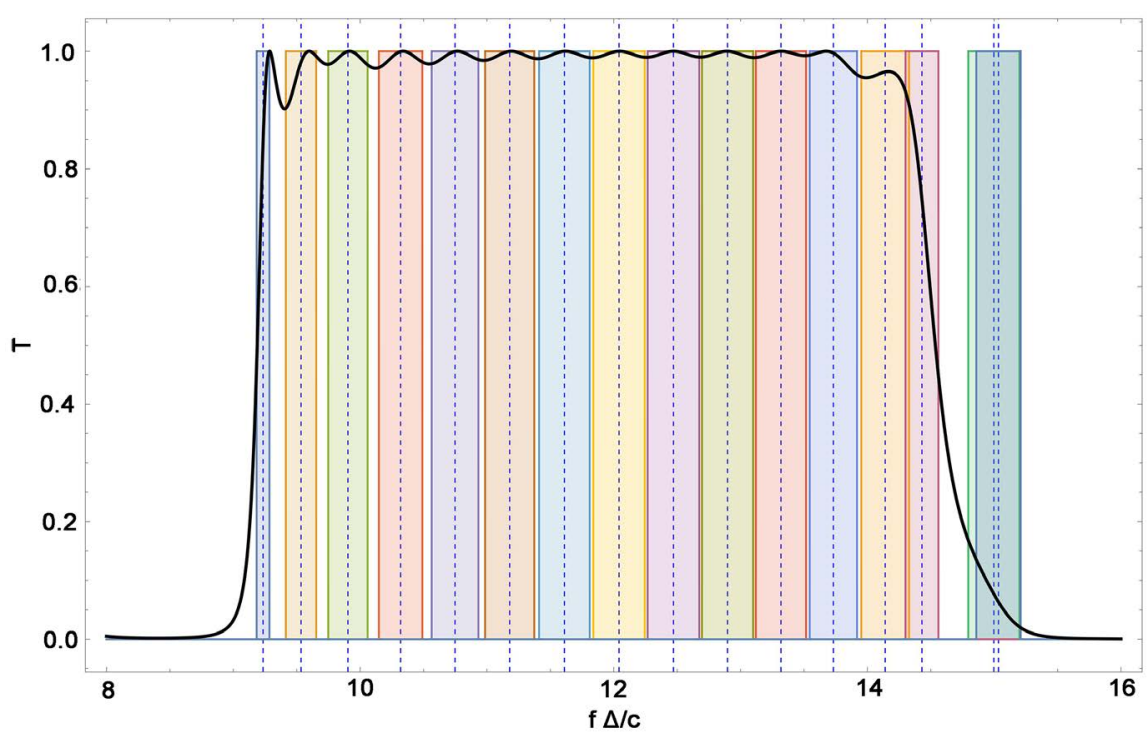

(a)

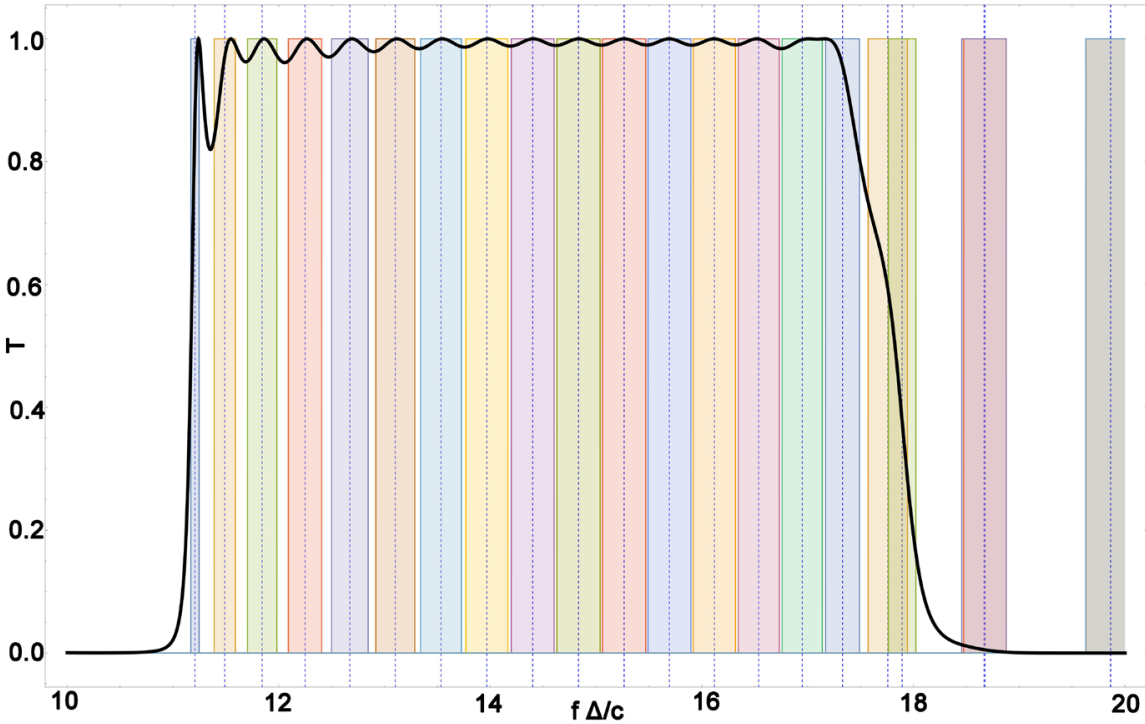

(b)

Figure 3. Transmission coefficient of for systems. The refractive index of odd layers of the structure are modulated by a Gaussian function. The parameters in such a system are: $n_{0}=n_{s}=n_{p}=1, n_{\max }=2$ and $d_{p}=8 d_{\text {odd }}$. The blue dashed lines correspond to the real part of the solution of the escape problem while the rectangles centered on each of them represent their corresponding $\Gamma$. (a) $n_{\min }=1.184$ and $N_{c}=37$; (b) $n_{\min }=1.184$ and $N_{c}=45$.

We made the calculations using Mathematica 10.4. It is important to stress that it was difficult to solve the escape problem in (10) for more than 11 layers. This was overcome separating the element matrix $M_{11}$ equals to zero in its real and imaginary part and using an interpolation with known numerical methods.

From Figures 2-4 we see that the Gaussian systems present transparency bands with gaps of no transmission. For these systems the escape states does not necessarily coincide with the resonaces of the passbands and the escape states 


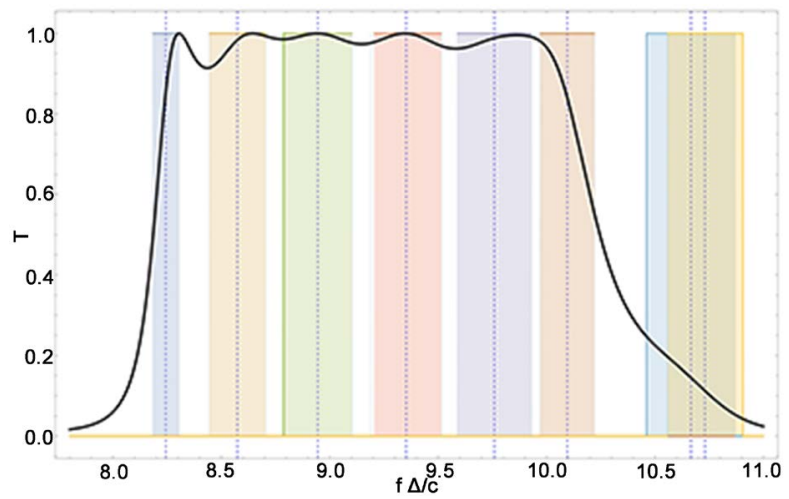

(a)

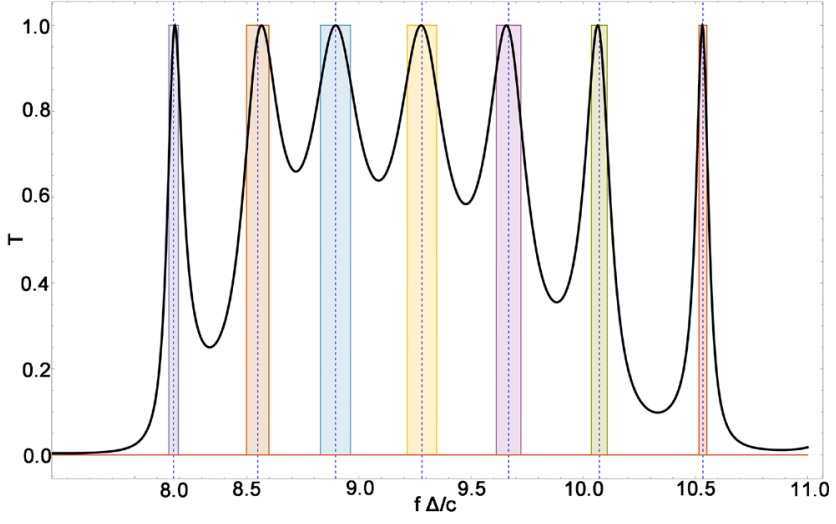

(b)

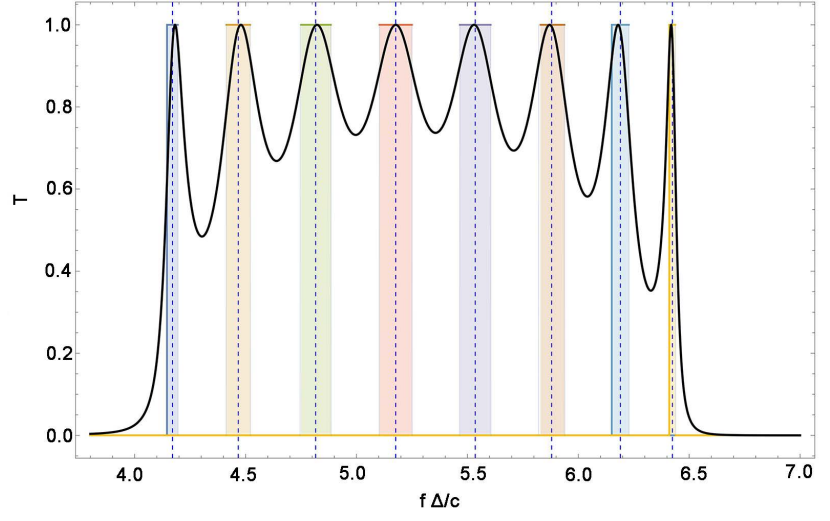

(c)

Figure 4. Comparison between the system modulated by a Gaussian function and the regular with 17 layers. Parameters in such a system are: $n_{0}=n_{s}=n_{p}=1, n_{\max }=2$, and $d_{p}=8 d_{\text {odd }}$. The blue dashed lines correspond to the real part of the solution to the escape problem while the rectangles centered on each of them represent their corresponding $\Gamma$. (a) System where odd layers are modulated by a Gaussian function so that the minimum refractive index is at the ends and the largest at the center of the structure; (b) System where the odd layers are modulated by a Gaussian function so that the maximum refractive index is at the ends and the minimum at the center of the structure; (c) Regular system, i.e., the value of the refractive index in the odd layers is constant and equal, $n=2$.

are very wide. By contrast, the reversed Gaussian and the regular structures present passbands without transparency, only peaks of resonances of transmission for the eigenstates of the system. At the same time, for these structures, the coincidence of escape states and resonances of transmission is much better and the escape states are narrow. This means that the escape states lifetime for Gaussian systems is shorter than for a reversed Gaussian and regular systems, which is what we expected because the photons are more difficult to escape from these last systems. By contrast, the progressive reduction of refractive index from the middle of the structure to the extremes, facilitates the escape of photons in a Gaussian system . Simultaneously, from the transmission bands and the position of the wide escape states for Gaussian structures, we deduce that the transparency bands are formed by the envelope of the wide escape states, which was the expectation mentioned in the Introduction. Mathematically speaking we are facing two different boundary problems on the same equation. The solutions 
need not be the same. In fact, the transmission problem has a continuum spectrum while the escape problem has a discrete spectrum with complex values. However, in certain situations, as the case of the regular structure, the two problems in some extent appear to be closely related. In the case of the Gaussian structure the relationship between the two problems is less clear.

\section{Conclusions}

Using the Maxwell's equation and the transfer matrix formalism, we obtained transmission coefficients and the solution of the problem of escape in systems where the refractive index of the layers is modulated by a Gaussian function, and for a reversed Gaussian and a regular systems. The variation of parameters such as the refractive index and the number of layers in Gaussian structures, allow passbands with very good transmission, i.e., very good transparency. This type of systems has potential applications as frequency filters for photons.

The structure has broad intervals of frequency or passbands where there is almost total transmission, separated by stopbands where there is no propagation of photons. The escape frequencies have very wide linewidths and are situated inside or near the passbands, but they do not necessarily coincide with the passbands. For Gaussian structures, the escape frequencies have very wide linewidths and are situated inside or near the passbands, but they do not necessarily coincide with the passbands. For a regular and and inverted Gaussian structures the resonance and the escape frequencies are very close. We associate the large width of the escape frequencies to the formation of transparency bands in the Gaussian structures.

\section{Acknowledgements}

MLSV and DACS thank the support of PRODEP SEP-SES. CIC wishes to thank the support of COZCYT and also of CONACYT (Grant Number 337137). RPA acknowledges hospitality at Autonomous University of Zacatecas.

\section{References}

[1] Yablonovich, Y. (1987) Inhibited Spontaneous Emission in Solid-State Physics and Electronics. Physical Review Letters, 58, 2059-2062. https://doi.org/10.1103/PhysRevLett.58.2059

[2] ohn, S. (1987) Strong Localization of Photons in Certain Disordered Dielectric Superlattices. Physical Review Letters, 58, 2486-2489. https://doi.org/10.1103/PhysRevLett.58.2486

[3] O’Brien, J.D., Lee, P., Cao, J.R., Kuang, W., Kim, C., Kim, W., Yang, T. and Choi, S. (2004) Photonic Crystal Lasers. ENN, 8, 617-628.

[4] Istrate, E. and Sargent, E.H. (2006) Photonic Crystal Heterostructures and Interfaces. Reviews of Modern Physics, 78, 455-481. https://doi.org/10.1103/RevModPhys.78.455

[5] Joannopoulos, J.D., Johnson, S.G., Winn, J.N. and Meade, R.D. (2008) Photonic Crystals: Molding the Flow of Light. Princeton University Press, Princeton. 
[6] Aly, A.H., Ismaeel, M. and Abdel-Rahman, E. (2012) Comparative Study of the One Dimensional Dielectric and Metallic Photonic Crystals. Optics and Photonocs Journal, 2, 105-112. https://doi.org/10.4236/opj.2012.22014

[7] Segovia-Chaves, F. (2014) Energy Flux Reflected and Transmitted in One-Dimensional Photonic Crystal. Revista de la Facultad de Ciencias Básicas, 10, 158-167. https://doi.org/10.18359/rfcb.327

[8] Madrigal-Melchor, J., Enciso-Muñoz, A. and Contreras-Solorio, D.A. (2013) Optical Transmittance of a Multilayer Structure with Gaussian Modulation of the Refractive Index. IOP Conference Series. Materials Science and Engineering, 45, 012032. http://iopscience.iop.org/article/10.1088/1757-899X/45/1/012032 https://doi.org/10.1088/1757-899X/45/1/012032

[9] Maksimović, M. (2008) Optical Resonances in Multilayer Structures. PhD Thesis, University of Twente, The Netherlands.

[10] Settimi, A., Severini, S., Mattiucci, N., Sibilia, C., Centini, M., D’Aguanno, G. and Bertolotti, M., Scalora, M., Bloemer, M. and Bowden, C.M. (2013) Quasinormal-Mode Description of Waves in One-Dimensional Photonic Crystals. Physical Review E, 68, 026614. https://doi.org/10.1103/PhysRevE.68.026614

[11] Silba-Vélez, M. de la Luz, Pérez-Álvarez, R. and Contreras-Solorio, D.A. (2015) Transmission and Escape in Finite Superlattices with Gaussian Modulation. Revista Mexicana de Física, 61, 132-136.

[12] Pérez-Alvarez, R. and Rodriguez-Coppola, H. (1988) Transfer Matrix in 1D Schrödinger Problems with Constant and Position-Dependent Mass. Physica Status Solidi B, 145, 493-500. https://doi.org/10.1002/pssb.2221450214

[13] Pérez-Álvarez, R., Trallero-Herrero, C. and Garca-Moliner, F. (2001) Transfer Matrix in One Dimensional Problems. European Journal of Physics, 22, 275. https://doi.org/10.1088/0143-0807/22/4/302

[14] Pérez-Álvarez, R. and Garca-Moliner, F. (2004) Transfer Matrix, Green Function and Related Techniques: Tools for the Study of Multilayer Heterostructures. Universitat Jaume I.

[15] Silba-Vélez, M. de la Luz (2010) One-Dimensional Variable Mass Problems in Multilayer Systems. Thesis, Faculty of Sciences, Autonomous University of the State of Morelos, Cuernavaca, Mexico.

[16] Pochi Yeh (2005) Optical Waves in Layered Media. 2nd Edition, Wiley-Interscience, Hoboken.

[17] Luna, H.R. (2007) Transmittance of a Layer with Negative Refractive Index. Masters Thesis, Academic Unit of Physics, Autonomous University of Zacatecas, Zacatecas. 